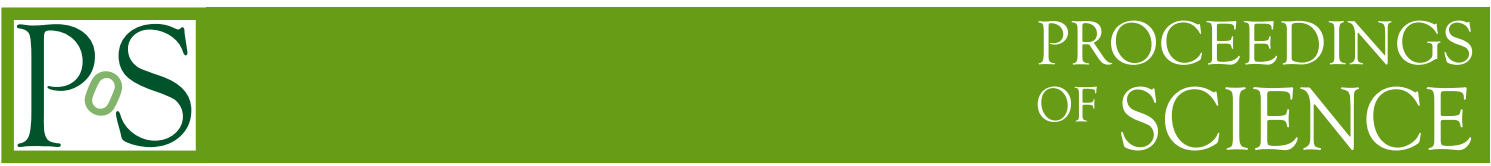

\title{
"Realistic" point source maps at Planck frequencies
}

\author{
Marcella Massardi* \\ SISSA/ISAS \\ Via Beirut 2-4, I-34014 Trieste, Italy \\ E-mail: massardiesissa.it \\ Joaquin Gonzalez-Nuevo \\ SISSA/ISAS \\ Via Beirut 2-4, I-34014 Trieste, Italy \\ E-mail: gnuevo@sissa.it
}

\section{Gianfranco De Zotti}

INAF-Osservatorio Astronomico di Padova

Vicolo dell'Osservatorio 5, I-35122 Padova, Italy

E-mail: dezotti@pd.astro.it

\begin{abstract}
We present a set of realistic all-sky maps of radio and IR sources based on existing catalogues, extrapolated to Planck frequencies. The observational information on spectral behaviour and polarization properties of sources has been exploited. Up to date models have been used to extend the maps beyond the flux limits of available surveys. Our maps could be very useful for testing the efficiency of source extraction and component separation algorithms as well as to assess the prospects for detecting primordial non-Gaussianities in CMB maps in the presence of point source contamination.
\end{abstract}

$C M B$ and Physics of the Early Universe

20-22 April 2006

Ischia, Italy

\footnotetext{
* Speaker.
} 


\section{Radio Sources}

A summary of the available relatively deep multi-steradian surveys is given in the Table 1 . The combined $4.85 \mathrm{GHz}$ GB6 and PMN surveys cover the whole sky (except for $0.21 \mathrm{sr}$ in the Northern polar cap and a tiny region around the Southern celestial pole) with an angular resolution of 3.5' $-4.2^{\prime}$ and a flux limit ranging from 18 to $72 \mathrm{mJy}$. Deeper and higher resolution surveys have been carried out at 1.4 and $0.843 \mathrm{GHz}$.

\begin{tabular}{|c|c|c|c|c|}
\hline Frequency & Catalogue & $S_{\min }(\mathrm{mJy})$ & Dec $_{\text {lim }}$ & Resolution \\
\hline $4.85 \mathrm{GHz}$ & GB6 & 18 & $0 \div+75$ & 3.5 \\
& PMNS & 20 & $-87.5 \div-37$ & $4.2^{\prime}$ \\
& PMNZ & 72 & $-37 \div-29$ & $4.2^{\prime}$ \\
& PMNT & 42 & $-29 \div-9.5$ & $4.2^{\prime}$ \\
& PMNE & 40 & $-9.5 \div+10$ & $4.2^{\prime}$ \\
\hline $1.4 \mathrm{GHz}$ & NVSS & 2.5 & $-40 \div+90$ & $45^{\prime}$ \\
\hline $0.843 \mathrm{GHz}$ & SUMSS & $8-18$ & $-90 \div-30$ & 45 'cosec $\mid$ dec \\
\hline
\end{tabular}

Table 1: A summary of the available relatively deep multi-steradian surveys

The cross-correlations of catalogues at different frequencies have been carried out degrading the higher resolution one to the resolution of the other. In practice, whenever the higher resolution (NVSS or SUMSS) catalogue contains more than one source within the resolution element of the lower resolution $(4.85 \mathrm{GHz})$ one, we have summed the NVSS or SUMSS fluxes, weighted with a Gaussian response function centered on the nominal position of the $4.85 \mathrm{GHz}$ source, and with FWHM equal to the resolution of the $4.85 \mathrm{GHz}$ survey. To correct for the contributions of background sources we have selected 159,195 control fields, free of $4.85 \mathrm{GHz}$ sources, and computed the average flux of NVSS or SUMSS sources within a $4.85 \mathrm{GHz}$ beam pointing on the field center, again taking into account the $4.85 \mathrm{GHz}$ response function. Such average flux has been subtracted from the summed NVSS or SUMSS fluxes associated with $4.85 \mathrm{GHz}$ sources. In this way we obtained spectral indices from $\sim 1$ to $\sim 5 \mathrm{GHz}$ for a complete sample (CCS sample) of 109,152 sources over about $95 \%$ of the sky to a flux limit of $\sim 50 \mathrm{mJy}$. The main holes of the CCS sample are in the North Pole region (due to the GB6 coverage) and on half of the Galactic plane (caused by the SUMSS coverage).

Positions and $1 \mathrm{GHz}$ fluxes only are available, from NVSS+SUMSS, for a deeper sample of $1,980,491$ sources brighter than $\sim 5 \mathrm{mJy}$. The hole in the SUMSS survey is partially filled by the $4.85 \mathrm{GHz}$ PMNS survey, which however is limited to $40 \mathrm{mJy}$. To build a spatially homogeneous map we randomly copied into this region sources with lower fluxes from other regions until the mean surface density as a function of the $\sim 1 \mathrm{GHz}$ flux equalled the overall mean down to $\sim 5$ mJy. In this way we obtained a uniform map over $100 \%$ of the sky, with positions of real sources, except for a minor fraction $(\sim 4 \%)$ of simulated sources. Note that the latter are located in the Galactic plane region, where they make a minor effect compared to Galactic emissions and have flux densities well below those relevant for Planck. We have estimated spectral indices, $\alpha$ ( $S \propto$ $v^{-\alpha}$ ), of sources with flux measurements at two frequencies, and have classified them as "flat"- or "steep"- spectrum for $\alpha<$ or $>0.5$, respectively. The mean spectral indices of the two classes were 

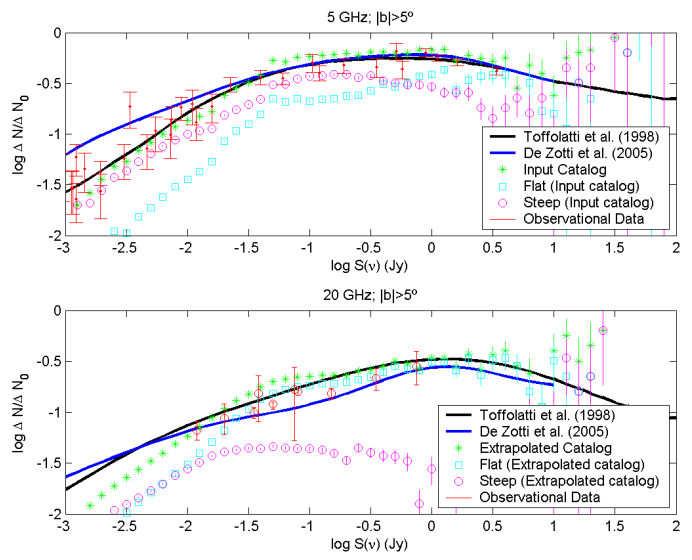

Figure 1: Source number counts at 5 and $20 \mathrm{GHz}$ compared with models and observational data.

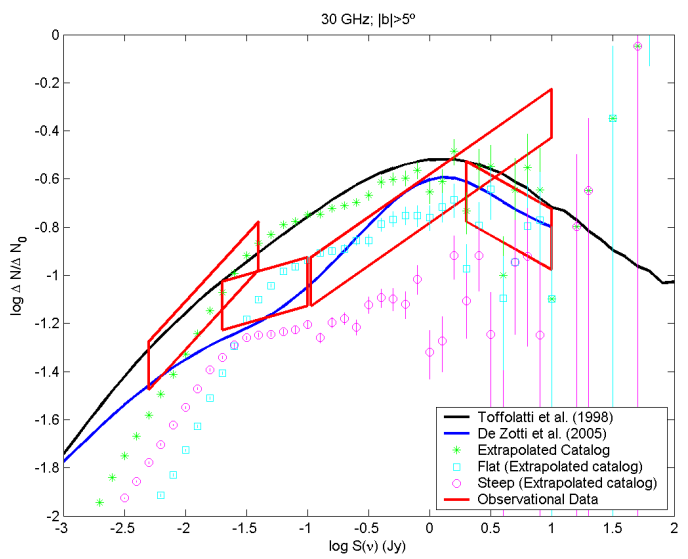

Figure 2: Source number counts at $30 \mathrm{GHz}$ compared with models and observational data.

found to be $\left\langle\alpha_{\text {flat }}\right\rangle=0.16,\left\langle\alpha_{\text {steep }}\right\rangle=1.18$. The dispersions round the mean values are $\sigma=0.3$ for both classes (Ricci et al. 2004).

We have assigned to each source a spectral index randomly drawn from a Gaussian distribution with the mean and variance of its class. Sources with measurements at a single frequency have been randomly assigned to the "flat"- or to the "steep"- spectrum class in the proportions observationally determined by Fomalont et al. (1991) for various flux intervals. Again, spectral indices have been randomly assigned drawing them from the above Gaussian distributions. In this way we have built simulated maps up to $20 \mathrm{GHz}$. The forthcoming results of the ATG20 survey will allow us to substantially improve such maps. At the moment, we could only check that the counts derived from the simulated maps agree with the observed ones, as shown by Fig. 1. Due to the complex spectral shape of radio sources, the power-law approximation holds only for a limited frequency range. To extrapolate the fluxes to still higher frequencies we used the multifrequency WMAP data (Bennett et al. 2003) to derive the distributions of differences da between spectral indices 


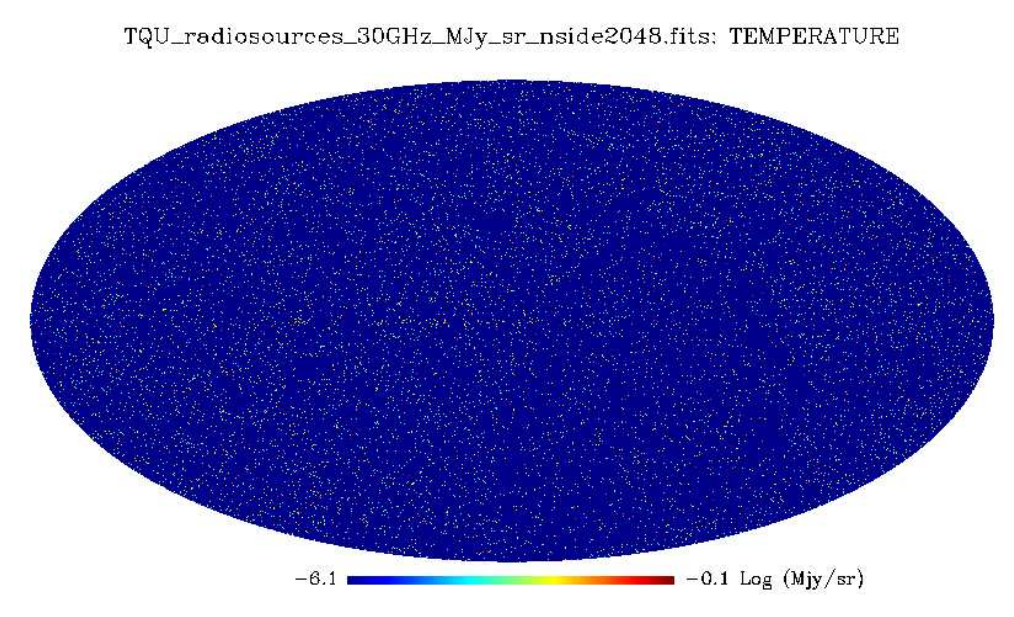

Figure 3: An all-sky simulation of radiosources at $30 \mathrm{GHz}$ in MJy/sr.

above and below $20 \mathrm{GHz}$. Such distributions can be approximated by Gaussians with mean 0.35 and dispersion 0.3. To each source we have associated a spectral index change drawn at random from the distribution. In Fig. 2 we compare the number counts calculated from the extrapolated catalogue with data from different surveys and with model predictions.

We have also produced maps of polarized emission attributing to each source a polarization degree randomly drawn from the observed distributions for flat- and steep-spectrum sources at 20 $\mathrm{GHz}$ (Ricci et al. 2004), and a polarization angle randomly drawn from a uniform distribution.

\section{Far-Infrared Sources}

Combining far-IR (IRAS) and sub-mm (from the SCUBA Local Universe Galaxy Survey) measurements Serjeant \& Harrison (2005) interpolated and extrapolated the IRAS detections to make predictions of the SEDs of all 15411 PSC-z galaxies from 50-1300 microns. A compilation including all FIR sources taken from the IRAS PSS and FSC catalogues was made available to the Planck WG2 by Dave Clements. As the PSC does not contain objects where the confusion from Galactic sources is high, and thus does not penetrate the Galactic centre well, and the FSC is restricted to regions away from the Galactic plane, the source density is a function of Galactic latitude. We have complemented the Serjeant \& Harrison's catalogue with the additional IRAS sources in Clements' compilation. In this way we obtained a source catalogue which looks approximately complete down to $\sim 80 \mathrm{mJy}$ at $857 \mathrm{GHz}$ (see Fig. 4). The coverage gaps of the catalog were filled by adding randomly distributed sources until the mean surface density as a function of flux matched the mean of well covered regions.

On the other hand, an important, and possibly dominant, contribution to small-scale anisotropies comes from galaxies selected by (sub)-mm surveys with SCUBA and MAMBO, that are probably strongly clustered (Negrello et al. 2004). These galaxies are interpreted as massive protospheroidal galaxies in the process of forming most of their stars in a gigantic starburst. We have adopted the counts predicted by the Granato et al. (2004) model, which successfully accounts for 


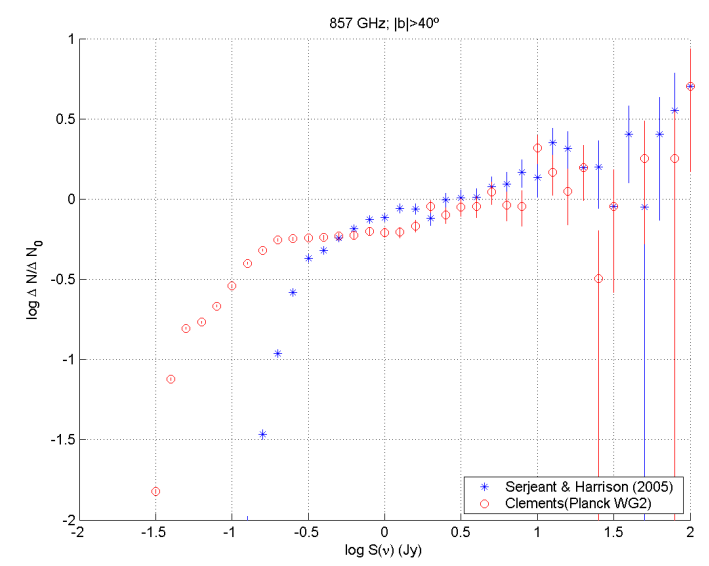

Figure 4: Comparison between the source number counts of Serjeant \& Harrison (2005) and the Clements ones at $857 \mathrm{GHz}$ for a galactic cut of $|b|>40$.

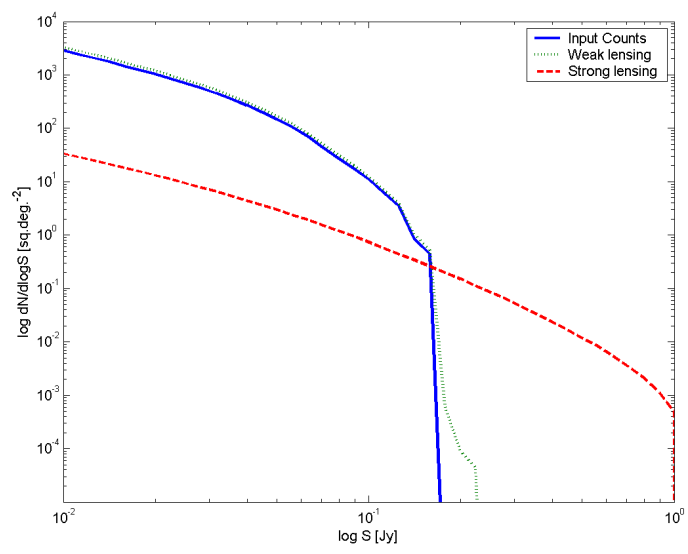

Figure 5: Predicted counts of strong (red dashed line) and weak (green dotted line) lensed sources of Perrotta et al.(2003) compared with the source number count of Granato et al.(2004) model.

a broad variety of data including the SCUBA and MAMBO counts and the preliminary redshift distribution (Chapman et al. 2005). The clustering properties of these sources have been modelled as in Negrello et al. (2005). The simulation of their spatial distribution has been produced using the method by Gonzalez-Nuevo et al. (2005). Once a map of the source distribution was obtained at the reference frequency, $v_{r e f}$, extrapolations to any other frequency, $v_{i}$, were obtained via the spectral indices $\alpha=-\log \left(S_{\text {ref }} / S_{i}\right) / \log \left(v_{\text {ref }} / v_{i}\right)$, were $S_{i}$ is defined by $n\left(>S_{i} ; v_{i}\right)=n\left(>S_{\text {ref }} ; v_{r e f}\right)$. The spectral indices have been computed in logarithmic steps of 0.1 . We have checked that the counts computed from the extrapolated maps accurately match those yielded, at each frequency, by the model.

Finally, Perrotta et al. (2003) found that the fraction of gravitational lensed sources at bright $\mathrm{mm} / \mathrm{sub}-\mathrm{mm}$ sources is expected to be up to $40 \%$. Using their predicted counts of strong lensed 


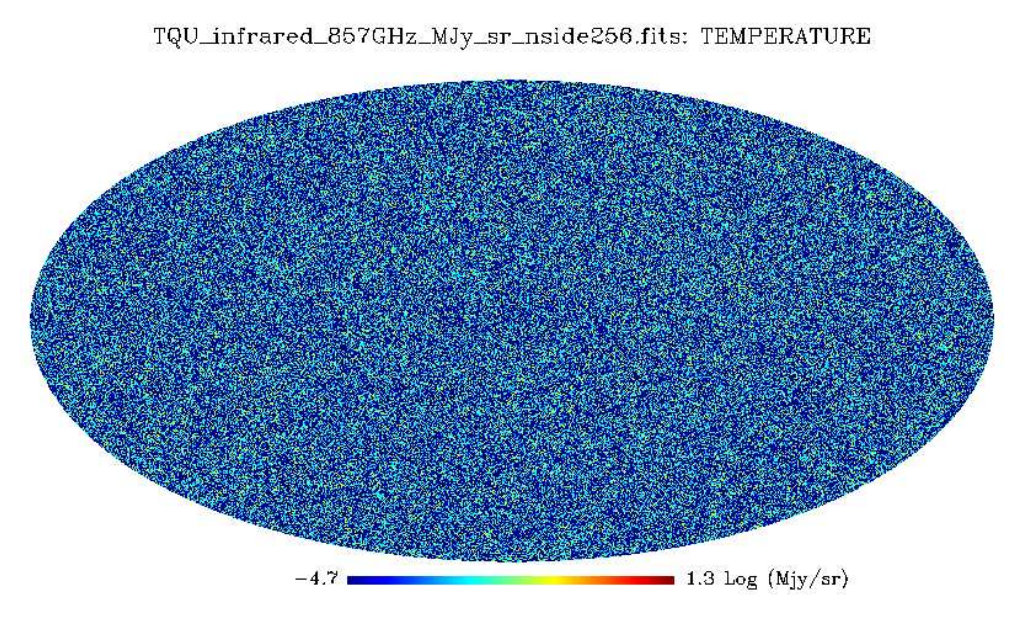

Figure 6: An all-sky simulation of FIR sources at $857 \mathrm{GHz}$ in MJy/sr.

sources (see Fig. 5), we have simulated the sources following a Poisson distribution. The frequency extrapolations were made via the spectral indices obtained in the same way as for the proto-spheroidal galaxies.

To each source we have assigned a polarization degree of $1 \%$ and a polarization angle randomly drawn from a uniform distribution.

\section{References}

[1] Bennet C.L. et al., 2003, ApJS, 148, 97-117.

[2] Chapman S.C. et al., 2005, ApJ, 622, 772-796.

[3] De Zotti G. et al, 2005, A\&A, 431, 893-903.

[4] Fomalont E.B. et al., 1991, AJ,102, 1258-1277.

[5] Gonzalez-Nuevo J.,Toffolatti L., Argueso F., 2005,ApJ, 621, 1-14.

[6] Granato G. et al., 2004, ApJ, 600, 580-594.

[7] Negrello M. et al., 2004, MNRAS, 352, 493-500.

[8] Perrotta F. et al., 2003, MNRAS, 338, 623-636.

[9] Ricci R. et al., 2004, MNRAS, 354, 305-320.

[10] Ricci R. et al., 2004, A\&A, 415, 549-558.

[11] Serjeant S., Harrison D., 2005, MNRAS, 356, 192-204.

[12] Toffolatti L. et al., 1998, MNRAS, 297, 117-127. 